\title{
Influencia de la técnica de soldadura multipasada y de los tratamientos térmicos de precalentamiento y post-soldadura en el comportamiento de uniones GMAW de un acero microaleado HARDOX 400(*)
}

\author{
A. Martínez*, V. Miguel**, J. Coello*, A. Navarro**, A. Calatayud** y M.C. Manjabacas**
}

\begin{abstract}
Resumen
Los aceros microaleados pueden suministrarse endurecidos. En estos casos, aunque el acero sea soldable, puede dar lugar a índices de soldabilidad que pueden ser mejorables con tratamientos térmicos de precalentamiento y/o tratamientos post-soldeo. En el presente trabajo se analiza el efecto del precalentamiento, soldeo multipasada y postcalentamiento en uniones GMAW del acero HARDOX 400. Se realizan análisis macro y microestructurales de las zonas características de la unión y del comportamiento mecánico de la misma en función de las variables indicadas. Los resultados obtenidos indican el efecto beneficioso del precalentamiento debido al alejamiento de la zona de menor dureza en la ZAT respecto al borde del cordón. También se demuestra que el precalentamiento aumenta el índice de heterogeneidad de la unión, lo que mejora su comportamiento a fatiga, tal y como demuestran algunos autores. El tratamiento térmico postsoldeo también presenta un efecto beneficioso ya que refuerza ligeramente la zona de menor dureza de la ZAT y favorece la plasticidad en la rotura. El efecto beneficioso del tratamiento postsoldeo es mayor en uniones soldadas con precalentamiento. La soldadura multipasada no presenta ninguna mejora destacable frente al soldeo en pasada única.
\end{abstract}

\section{Analisys of the influence of the multipass welding, welding preaheat and welding post heat treatments on the behaviour of GMAW joints of HARDOX 400 microalloyed steel}

\begin{abstract}
The microalloyed steels may be supplied in a hardened state. In these cases, the weldability can be improved by pre-heat and/or post-heat welding treatments. In this paper, the effect of those treatments and the influence of multipass welding on GMAW joints behavior are analyzed for a Hardox 400 microalloyed steel. The microstructure evaluation of the different heat affected zones of the steel has been made and the mechanical properties of those zones are obtained for different conditions as it has been mentioned. The obtained results indicate that preheating the steel leads to a beneficial action that consists on the distance increasing from the bead to the zone in which the hardness is lowest. The postheat treatment strengthens that zone and improves the joint plasticity. This benefit is higher if the joint has been made with preheating. Multipass welding has not been found to have any advantage if it is compared to a single welding pass.
\end{abstract}

Keywords Weldability; Hardox 400; Preheating; Welding postheating; Microalloyed steel.

\section{INTRODUCCIÓN}

Los aceros microaleados, también conocidos como aceros HSLA (High Strength Low Alloy Steels) ó de alta resistencia y baja aleación, son aceros estructurales de bajo contenido en carbono, generalmente inferior al 0,20\%, que contienen en su composición química pequeñas cantidades de elementos de aleación, generalmente inferiores al 0,15\%, formadores de carburos, nitruros y carbonitruros, con los que se controla las propiedades del material. El abanico de composiciones químicas es bastante amplio y la

(•) Trabajo recibido el día 28 de Enero de 2010 y aceptado en su forma final el día 27 de Julio de 2010.

* Instituto de Desarrollo Regional, Universidad de Castilla-La Mancha, Avda. España s/n 02071 Albacete.

** Escuela de Ingenieros Industriales, Universidad de Castilla-La Mancha, Avda. España s/n 02071 Albacete. 
identificación del acero en ocasiones suele corresponderse con denominaciones comerciales que establecen los diversos fabricantes.

Estos aceros están sujetos a diversos tratamientos de endurecimiento que se pueden llevar a cabo en el momento de la laminación en caliente. El procesado termomecánico conduce a microestructuras de escala fina que aseguran la alta resistencia y la tenacidad sin necesidad de emplear elementos de aleación que pongan en peligro la soldabilidad del material. Se ha demostrado que una microestructura bainítica con bajo contenido en carbono es una alternativa para los objetivos indicados ${ }^{[1]}$.

Otros procedimientos de endurecimiento suelen corresponderse con la fosforización de soluciones sólidas y el endurecimiento parcial mediante la dispersión de partículas de martensita duras en una matriz ferrítica $^{[2]}$. Esto último da lugar a los aceros dúplex o de doble fase y suelen tener un interés especial para aplicaciones en vehículos, dada la combinación de resistencia y conformabilidad. Particularmente, en estructuras o volquetes de vehículos industriales es adecuado el empleo de chapa gruesa de acero de alta resistencia que permita reducir el peso de aquéllos, junto con características excelentes de tenacidad y de soldabilidad ${ }^{[3]}$.

Otras propiedades específicas pueden ser exigidas en función de la aplicación concreta del material. Una de las aplicaciones para las que se diseñan aceros microaleados está relacionada con una elevada resistencia al desgaste. Aunque la dureza del material no determina por sí misma el éxito de la aplicación, resulta una propiedad importante ${ }^{[4]}$. Si la dureza del acero es superior a la que tienen las partículas abrasivas que se ponen en contacto sobre la chapa en operaciones de servicio, éstas apenas pueden causar deformación plástica en el material. La deformación elástica y la energía transmitida al material dependen del módulo elástico. Cuando el módulo de elasticidad es elevado, la penetración elástica que tiene lugar es menor. Si la dureza del acero es inferior a la de las partículas erosivas, entonces se produce el desgaste del mismo, así como efectos ploughing y de microcorte con el consiguiente deterioro de la chapa. La combinación de todas las propiedades indicadas anteriormente puede ser interesante en aplicaciones de vehículos pensados para el transporte de áridos y elementos de cantera.

En este trabajo, se analiza el comportamiento mecánico de uniones de chapa de $6 \mathrm{~mm}$ de espesor de acero de denominación HARDOX 400 soldadas mediante el procedimiento GMAW, bajo condiciones industriales. El acero HARDOX 400 es concebido para aplicaciones en las que se requiere buenas características estructurales, conformabilidad y alta resistencia al desgaste $^{[5]}$. Se suministra con tratamiento de temple o de temple-revenido. La soldabilidad de este acero resulta adecuada por cuanto su composición conduce a contenidos, en términos de carbono equivalente, que lo hacen técnicamente soldable sin precauciones especiales. La zona afectada térmicamente se define por las alteraciones microestructurales producidas sobre el metal base. En este caso, dada la estructura de partida del acero, con estructuras típicas duras correspondientes al tratamiento térmico en el que se suministra por el fabricante, la zona afectada térmicamente se caracteriza por una menor dureza en relación al metal base. La diferencia de comportamiento puede favorecer fenómenos de rotura de la unión soldada, así como condicionar el comportamiento a fatiga ${ }^{[6]}$ y a fatiga por desgaste superficial[ ${ }^{[7]}$.

Concretamente, ha sido establecido experimentalmente con aceros similares al descrito que con una microestructura homogénea se obtiene un mejor comportamiento tanto frente al desgaste por erosión como al desgaste abrasivo. La presencia de constituyentes de distinta dureza en la microestructura del acero puede conducir también a empeorar el comportamiento frente al desgaste por erosión ${ }^{[8]}$.

En este tipo de uniones, consideradas de carácter no homogéneo debido al diferente comportamiento y microestructuras presentes entre el metal base y el cordón de soldadura, la diferencia de características resistentes condicionan el comportamiento a fatiga de la unión. La relación entre el límite elástico del cordón y del metal base establecen la razón de heterogeneidad o "mis-matched ratio", MMR. Cuando ésta es mayor de la unidad el comportamiento a fatiga de la unión soldada es mejor que en caso contrario ${ }^{[8 \text { y } 9]}$.

En relación con el proceso de soldeo del acero, el fabricante suele facilitar condiciones o precauciones especiales, en el caso que se requieran, con el fin de mejorar los resultados obtenibles. No obstante, en muchas ocasiones no es fácil seguir dichos consejos, especialmente en lo que se refiere a aplicación de tratamientos de precalentamiento y post-soldeo o al control de temperatura entre pasadas bajo especificaciones más o menos estrictas. Esto suele deberse a la diversidad de tamaños de las piezas a unir, lo que hace difícil el empleo de calentadores eléctricos y la aplicación de temperatura mediante otros métodos. También hay que reseñar condicionantes de tipo económico que suelen estar presentes en los procesos industriales en general.

Un ejemplo de fallo típico relacionado con lo anterior consiste en la fractura que tiene lugar en volquetes de transporte de áridos. La fractura se produce generalmente en la unión soldada del material base con los refuerzos, en el límite del cordón. Este tipo de ejecuciones se realiza, como se ha indicado anteriormente, mediante técnica GMAW con la deposición del cordón mediante una sola pasada y 
INFLUENCIA DE LA TÉCNICA DE SOLDADURA MULTIPASADA Y DE LOS TRATAMIENTOS TÉRMICOS DE PRECALENTAMENTO Y POST-SOLDADURA EN EL COMPORTAMIENTO DE UNIONES GMAW DE UN ACERO MICROALEADO HARDOX 400 ANALISYS OF THE INFLUENCE OF THE MULTIPASS WELDING, WELDNG PREAHEAT AND WELDING POST HEAT TREATMENTS ON THE BEHAVIOUR OF GMAW JONTS OF HARDOX 400 MICROALLYYED STEEL

sin precalentamiento. En casos excepcionales y para empresas con medios suficientes, se realiza un recocido de eliminación de tensiones de toda la estructura mediante un postcalentamiento en grandes hornos. Dicho proceso no es asequible para la mayoría de los fabricantes de volquetes. No obstante, la eficacia del tratamiento térmico postsoldeo es discutible, pudiendo ser sus efectos beneficiosos o perjudiciales desde el punto de vista del comportamiento en servicio de la unión ${ }^{[7]}$. El efecto del tratamiento depende de la composición química del acero, del procedimiento de unión empleado y del valor de los parámetros considerados del tratamiento térmico.

En base a lo indicado, se han seleccionado varios procedimientos sencillos de ejecución a nivel industrial que podrían modificar la zona afectada térmicamente hacia una distribución de durezas y microconstituyentes más idónea para resistir los esfuerzos y deformaciones e intentar reducir la susceptibilidad de las piezas al fallo por fatiga.

El objetivo del presente trabajo es la valoración del efecto térmico del soldeo sobre las características mecánicas y microestructurales de la chapa de acero HARDOX 400 de $6 \mathrm{~mm}$ de espesor para tres condiciones modificadoras de la secuencia clásica de soldeo: precalentamiento, postcalentamiento y soldeo multipasada.

\section{PROCEDIMIENTO EXPERIMENTAL}

\subsection{Análisis químico del material base}

La composición en carbono y azufre del acero HARDOX 400 fue determinada experimentalmente mediante un analizador C/S300 marca LECO. Para la determinación del contenido en carbono y azufre ha sido necesaria la extracción de dos muestras similares, de aproximadamente 1,0 g cada una, de la parte general de la chapa y alejada suficientemente de la unión soldada. La extracción de las muestras se ha realizado mediante corte metalográfico refrigerado. Posteriormente se han limpiado y desengrasado con agua jabonosa y acetona.

Para la determinación de los elementos metálicos se ha utilizado un fotoespectrómetro de emisión por plasma, OPTIMA 5300 DV, marca PERKIN ELMER.

Para ello se ha extraído una muestra de aproximadamente $0,2 \mathrm{~g}$ de una zona general de la chapa y alejada de la soldadura. La extracción se ha realizado mediante corte metalográfico refrigerado. Posteriormente se ha procedido a su limpieza y desengrase, digestión ácida en microondas, enrase hasta $50 \mathrm{ml}$ y análisis propiamente dicho.

\subsection{Obtención de muestras soldadas}

Se realizaron cupones de soldadura a partir de 2 chapas de $300 \times 150 \mathrm{~mm}^{2}$, mediante soldadura MIG (GMAW). Como material de aporte se utilizó hilo AWS A5.18 y la soldadura se ha realizado en condiciones industriales habituales para el espesor de chapa indicado, $6 \mathrm{~mm}$. El material de aporte fue seleccionado tomando como criterio la limitación de presencia de hidrógeno en la unión.

Se realizó el soldeo de las chapas sin preparación de bordes, con una separación mínima de aquéllas, por una sola cara y con penetración parcial (Fig. 1).
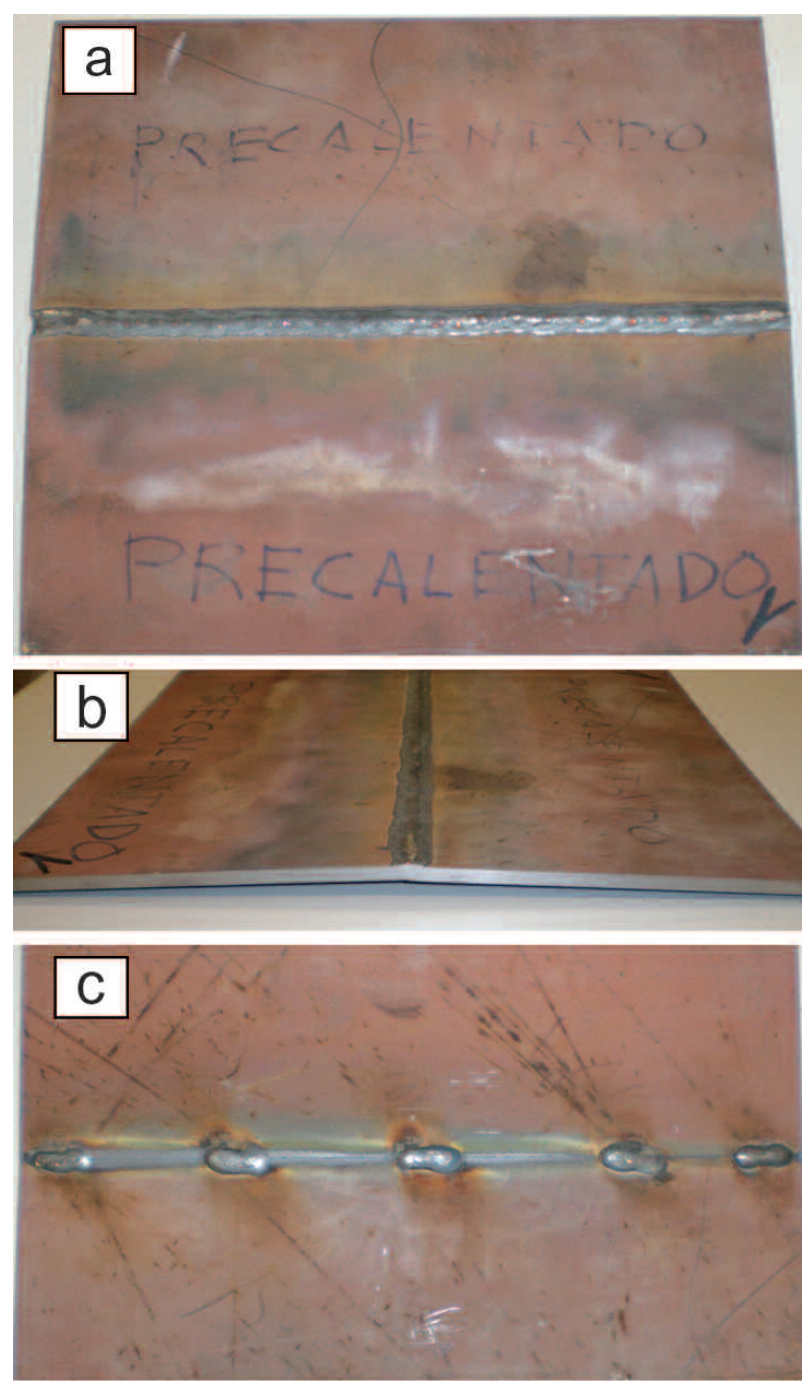

Figura 1. Cupón de soldadura con operación a una cara, sin penetración: a) cara del cordón de soldadura, b) sección transversal y c) cara de punteo para sujeción de las chapas.

Figure 1. Welding coupon for one side welding with no penetration: a) bead side, b) transversal section and c) welding points side. 
Se establecieron variaciones respecto a la operación de soldeo. Así se realizó la unión a cordón único por una cara con y sin precalentamiento de la chapa. También se realizó la operación mediante varios cordones o multipasada sin precalentamiento previo de la chapa. Las uniones fueron realizadas mediante punteo previo en una de las caras.

El precalentamiento se realizó mediante el empleo de un soplete butano-aire inmediatamente antes de soldar. La temperatura de la chapa precalentada mediante este método resultó estar alrededor de $200^{\circ} \mathrm{C}$. La determinación de la temperatura se llevó a cabo mediante medición con un termopar tipo p.

La operación se llevó a cabo sin y con precalentamiento antes del primer cordón de soldadura, (Fig. 2 a) y 2 b)) respectivamente, y mediante multipasada, (Fig. 2 c)).

\subsection{Ensayos macroestructurales, microestructurales y de microdureza}

De las muestras soldadas se extrajeron probetas metalográficas que abarcaban toda la soldadura, la zona
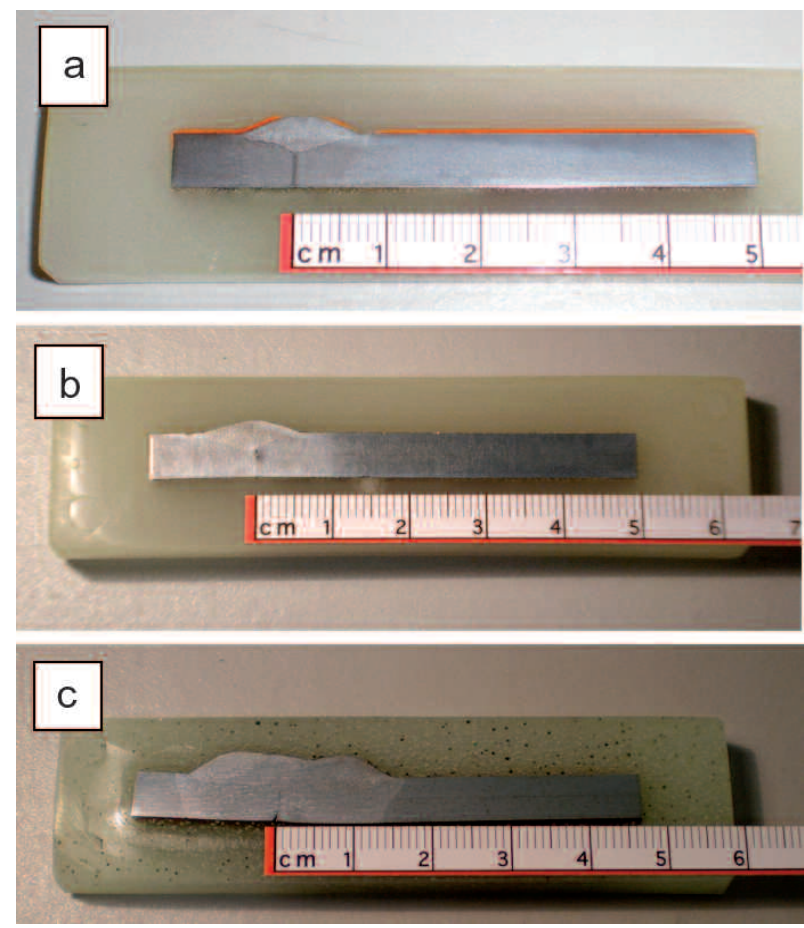

Figura 2. Uniones de acero Hardox 400 realizadas mediante soldadura MIG en chapas: a) sin precalentamiento, b) con precalentamiento y c) multipasada.

Figure 2. Hardox 400 butt joints welded by GMAW process: a) no preheatin, b) With preheating and c) multipass welding. afectada térmicamente (ZAT) y parte del material no afectado. La extracción se realizó mediante corte metalográfico refrigerado. Posteriormente se realizó la embutición con resina en frio, desbaste, pulido mecánico y ataque químico mediante Nital 1.

Los ensayos macroestructurales, se han realizado mediante la observación de las muestras en un proyector de perfiles y superficies NIKON V-12. Con dicho equipo se han valorado las superficies transversales de los cordones de soldadura en cada uno de los casos, distinguiendo entre área fundida bajo la superficie, Am y área sobre la misma, Ar, según se indica en la figura 3. La suma de ambas áreas supone el área total del material fundido, Aw. El parámetro de comparación entre los diferentes ensayos ha sido la relación porcentual entre áreas, C, expresada en la ecuación (1);

$$
C=\frac{A m}{A w} \times 100=\frac{A m}{A r+A m} \times 100
$$

La energía neta aportada a la pieza, Hnet, es normalmente expresada en función de la potencia eléctrica del arco, P, velocidad de soldeo, v, y un factor de eficiencia de transferencia de energía a la pieza, f1, conforme a la ecuación (2). La potencia eléctrica puede expresarse como el producto de la tensión y la intensidad del arco, V e I respectivamente.

$$
\text { Hnet }=f 1 \times \frac{P}{V}=f 1 \times \frac{V \times 1}{V}
$$

Puede demostrarse que, fijadas unas determinadas condiciones de soldeo, el área total de la parte fundida, Aw, es proporcional a la energía específica neta aportada ${ }^{[10]}$, tal y como se establece en la ecuación (3). En dicha ecuación f2 representa la eficiencia del proceso y Q la energía específica de fusión del material;

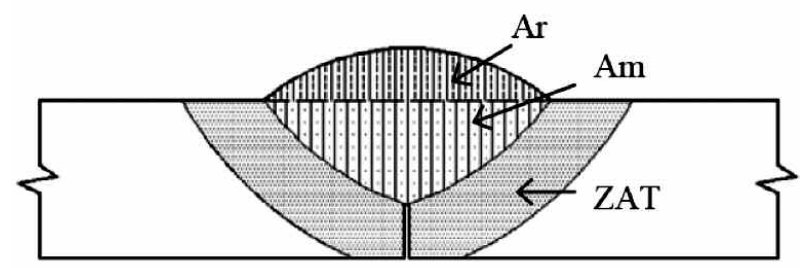

Figura 3. Diagrama de áreas de fusión en la sección del cordón de soldadura.

Figure 3. Diagram of fusion areas in the weld bead cross section. 


$$
A w=\frac{f 2 \times \text { Hnet }}{Q}=f 3 \times \text { Hnet }
$$

Según lo anterior, el índice de relación de áreas de fusión, C, en el cordón sirve de valor de comparación del efecto de las condiciones de contorno para diferentes procesos de soldadura, tales como la temperatura de precalentamiento de la chapa, espesor de chapa tamaño de los cupones y otros factores de influencia en la disipación de energía.

Los ensayos microestructurales se han realizado sobre las mismas muestras empleadas en el análisis macroscópico. Las observaciones microestructurales se han realizado mediante un microscopio metalográfico invertido NIKON EPIPHOT de hasta 1.000 aumentos.

Los ensayos de microdureza se han realizado sobre las mismas probetas con un microdurómetro SHIMADZU M. El método de ensayo utilizado ha sido de dureza Vickers a baja carga, con 300 g, conforme a lo establecido en la Norma UNE-EN-ISO 6507.

\subsection{Obtención de muestras para la realización de ensayos mecánicos}

Para estos ensayos fueron depositados sendos cordones de soldadura en ambas caras de una chapa de dimensiones $300 \times 300 \mathrm{~mm}^{2}$ con el fin de poder llevar a cabo ensayos mecánicos de tracción con las condiciones de simetría requeridas (Fig. 4). Las probetas consistieron en tiras de chapa de $150 \times 20 \mathrm{~mm}^{2}$ con la unión soldada en el medio. Aunque este tipo de probetas no es normalizado, el ensayo se ejecutó en las condiciones que indica la norma UNE EN 10002-1. Este tipo de soldadura pretende reproducir las condiciones que se producen en la soldadura de refuerzos a la chapa en aplicaciones de tipo estructural, como se ha indicado anteriormente, como por ejemplo para la fabricación de contenedores o bañeras para camiones de transporte de áridos. La operación se llevó a cabo sin y con precalentamiento antes del primer cordón de soldadura (Fig. 4 a) y 4 b)), respectivamente.

De las chapas así soldadas se extrajeron tiras de $20 \mathrm{~mm}$ perpendiculares al cordón para ensayos de tracción, de zonas suficientemente alejadas de los bordes.

Para los ensayos de resiliencia se obtuvieron tiras de $10 \mathrm{~mm}$ de anchura perpendiculares al cordón, tanto para el proceso con precalentamiento como sin él. Se eliminaron por mecanizado los excesos de los cordones y se tallaron las entallas. El tipo de entalla fue en $\mathrm{U}$, de $5 \mathrm{~mm}$ de profundidad y $2 \mathrm{~mm}$ de

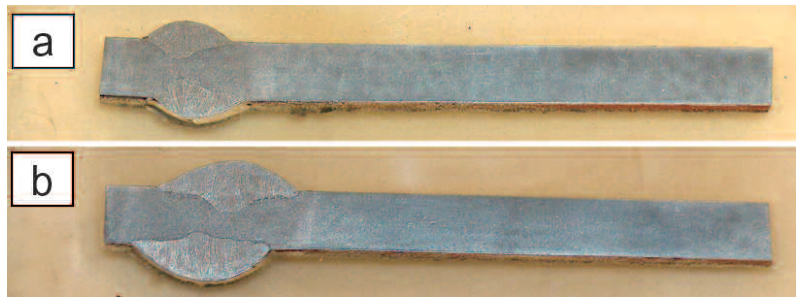

Figura 4. Detalle de cordones depositados sobre chapa Hardox 400 de dimensiones 300 x 300 x $6 \mathrm{~mm}^{3}$ : a) sin precalentamiento y b) con precalentamiento.

Figure 4. Some bead details (sheets size $300 \times 300 \times 6 \mathrm{~mm}^{3}$ ): a) no preheating and b) with preheating.

anchura (EN 10045-1). En cada grupo de probetas se realizaron las entallas en cuatro posiciones diferentes. Así, las entallas se localizaron en diferentes zonas conforme a las designaciones especificadas en la figura 5 y que se establecen como CC, en el centro del cordón de soldadura; EC, en un extremo del

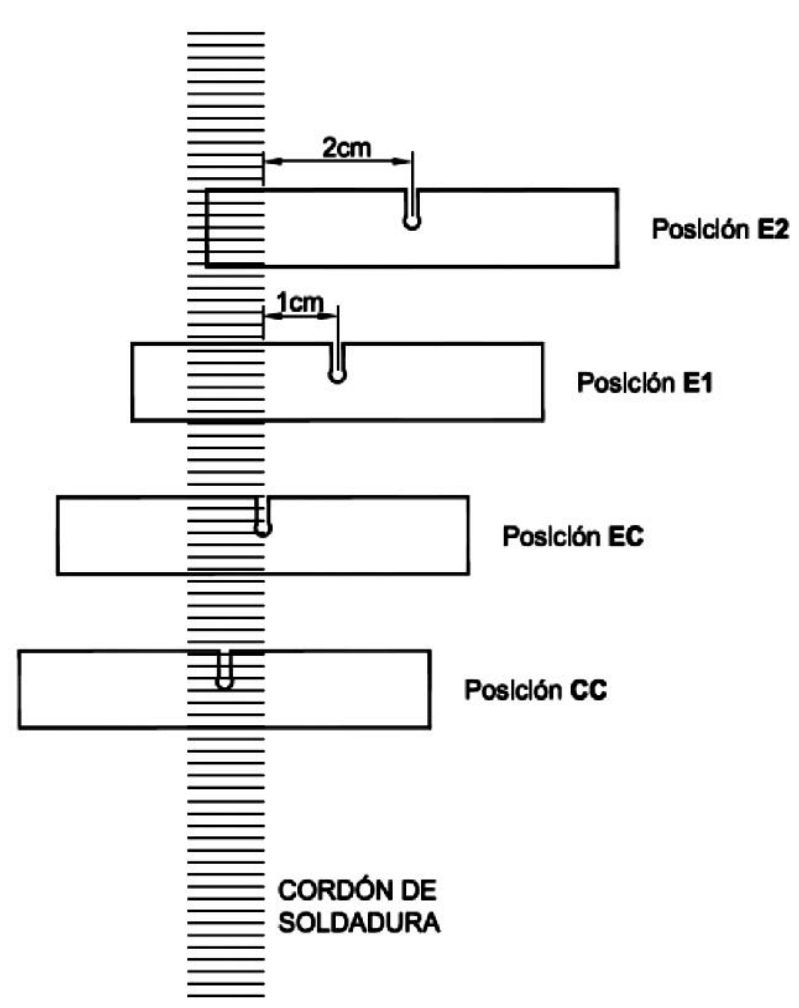

Figura 5. Designación de probetas para ensayos de resiliencia. Obtenidas a partir de secciones de $10 \times 6 \mathrm{~mm}^{2}$.

Figure 5. Resilience samples nomenclature. They are obtained from $10 \times 6 \mathrm{~mm}^{2}$ section size. 
cordón de soldadura; E1, a la distancia de $1 \mathrm{~cm}$ del extremo del cordón de soldadura; E2, a la distancia de $2 \mathrm{~cm}$ del extremo del cordón de soldadura. Aunque mayoritariamente se emplea una entalla tipo $\mathrm{V}$ en este tipo de ensayos, se ha seleccionado una entalla tipo $U$ debido a la mayor experiencia de los autores a la interpretación de los resultados con este tipo de entalla. En cualquier caso, existe una relación proporcional entre los valores obtenidos con ambos tipos de entalla ${ }^{[11]}$. También existe una correlación lineal entre los valores de resiliencia Charpy con entalla en $\mathrm{V}, \mathrm{CV}$, y los valores de apertura frente a la grieta, $\mathrm{CTOD}_{c,}{ }^{[12]} \mathrm{y}$, por consiguiente, una correlación con el factor de intensidad de tensiones crítico al cuadrado, $\mathrm{K}_{\mathrm{c}}^{2}$, para los ensayos de tenacidad a la fractura. Esto nos permite una estimación comparativa del comportamiento a la fractura para diferentes condiciones de soldeo y para diferentes posiciones de entalla con respecto a la soldadura.

Por tanto, los ensayos de resiliencia nos permiten estimar, al menos comparativamente, el comportamiento de diferentes procesos de soldadura en presencia de fisuras.

\section{RESULTADOS Y DISCUSIÓN}

\subsection{Análisis de composición del material base}

Los resultados de composición del material base aparecen en la tabla I.

\subsection{Análisis macrográfico de zonas de fusión}

Los resultados de las relaciones de áreas de fusión conforme a lo indicado en el epígrafe 2.3. se reflejan en la tabla II.

Como puede apreciarse, los valores con precalentamiento son superiores a los obtenidos sin él, como cabía esperar. La mayor temperatura de la chapa implica una menor disipación de la energía efectiva aportada y, por tanto, una mayor área fundida en función de la cantidad de material de aporte. De la misma manera, la soldadura multipasada posee una relación en el último cordón depositado similar a la soldadura con precalentamiento, por lo que cabría esperar efectos similares sobre la ZAT.

Para las soldaduras en ambas caras, el parámetro de velocidad de soldeo ha sido aumentado ligeramente, con el fin de conseguir que la relación de áreas en la segunda cara sea similar a las obtenidas en uniones a una sola cara, y que los resultados puedan analizarse de forma coherente. Aunque no se han conseguido obtener exactamente los mismos parámetros, los valores obtenidos de $\mathrm{C}$ para la soldadura de la segunda cara resultan comparables a las muestras soldadas por una sola cara en condiciones similares $(38,0$ frente a 45,8 y 50,1 frente a 58,4 ).

\subsection{Análisis microestructural}

En las figuras 6 y 7 se puede apreciar las microestructuras correspondientes al cordón de soldadura

Tabla I. Composición química del acero HARDOX 400 (\%)

Table I. Chemical composition of HARDOX 400 steel (\%)

\begin{tabular}{cccccccccccc}
\hline $\mathbf{C}$ & $\mathbf{S i}$ & $\mathbf{M n}$ & $\mathbf{P}$ & $\mathbf{S}$ & $\mathbf{C r}$ & $\mathbf{N i}$ & $\mathbf{M o}$ & $\mathbf{B}$ & $\mathbf{C o}$ & $\mathbf{C u}$ & $\mathbf{V}$ \\
\hline 0,12 & 0,35 & 1,08 & - & 0,003 & 0,24 & 0,05 & 0,014 & 0,004 & 0,063 & 0,014 & 0,014 \\
\hline
\end{tabular}

Tabla II. Relación de áreas fundidas, C, para diferentes condiciones de soldeo

Table II. Welded areas ratio, C, for different welding conditions

\begin{tabular}{ccccccc}
\hline \multicolumn{2}{c}{ SOLDADURA UNA CARA } & \multicolumn{3}{c}{ SOLDADURA AMBAS CARAS } \\
\hline \multicolumn{2}{c}{ Una pasada } & Multipasada & Sin Precal. & \multicolumn{2}{c}{ Precal. $200{ }^{\circ} \mathrm{C}$} \\
\hline $\begin{array}{c}\text { Sin Precal. } \\
45,8\end{array}$ & Precal. $200{ }^{\circ} \mathrm{C}$ & Sin Precal. & $1^{\text {a }}$ Cara & $2^{\text {a }}$ Cara & $1^{\text {a }}$ Cara & $2^{\text {a }}$ Cara \\
58,4 & 59,1 & 34,4 & 38,0 & 44,4 & 50,1 \\
\hline
\end{tabular}


INFLUENCIA DE LA TÉCNICA DE SOLDADURA MULTIPASADA Y DE LOS TRATAMENTOS TÉRMICOS DE PRECALENTAMENTO Y POST-SOLDADURA EN EL COMPORTAMENTO DE UNIONES GMAW DE UN ACERO MICROALEADO HARDOX 400 ANALISYS OF THE INFLUENCE OF THE MULTIPASS WELDING, WELDING PREAHEAT AND WELDNG POST HEAT TREATMENTS ON THE BEHAVIOUR OF GMAW JONTS OF HARDOX 400 MICROALLOYED STEEL

y a tres zonas diferenciadas de la ZAT. Esta circunstancia es común a todas las variaciones experimentadas en el soldeo. Lógicamente, la transición de unas zonas a otras se realiza de forma progresiva, por lo que existen pequeñas franjas en las que coexisten constituyentes de las zonas implicadas. La diferencia observada en relación a los diferentes procedimientos de soldeo se refleja únicamente en el tamaño de los constituyentes. La descripción de constituyentes se hace conforme a las especificaciones del Instituto Internacional de Soldadura, IIW [13]. El cordón de soldadura (Fig. 6 b)) refleja la cristalización típica columnar, con estructuras de ferrita primaria intergranular $\mathrm{PF}(\mathrm{G})$ junto con ferritas aciculares con segundas fases alineadas FS(A) envolviendo a una estructura general del tipo Widmanstätten, compuesta por ferritas con segundas fases no alineadas FS(NA) y martensita revenida, $M$.

La zona estructural 1 (Fig. 6 c)), se caracteriza por la presencia de una estructura de perlita fina $\mathrm{FC}$ en una matriz de ferrita primaria $\mathrm{PF}$ y ferrita con segunda fase alineada FS(A). Esta estructura es propia de un recocido con austenización completa.
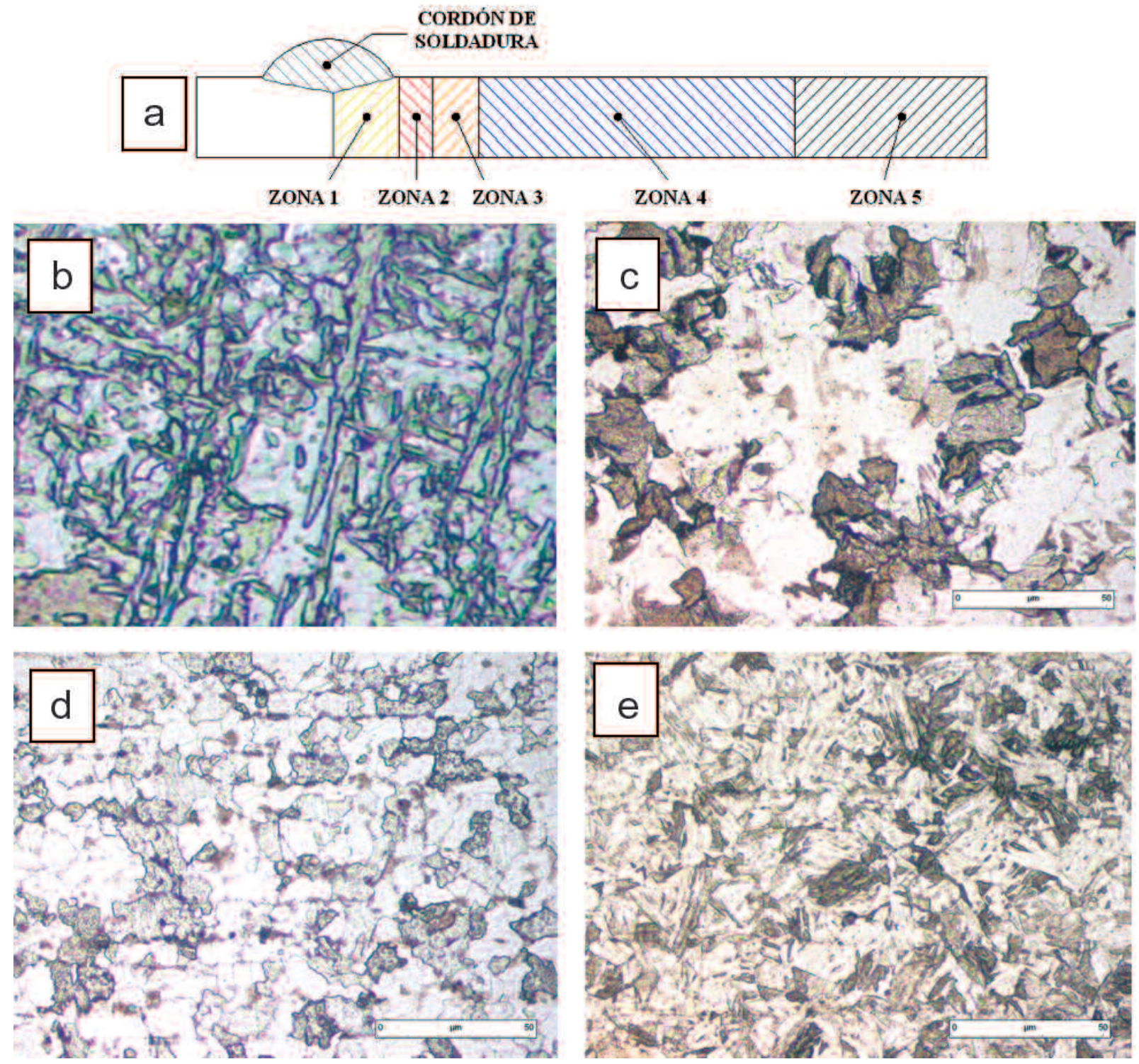

Figura 6. Microestructuras características de diversas zonas en la soldadura MIG del acero Hardox 400: a) esquema de las zonas existentes, b) cordón de soldadura, c) ZAT zona 1, d) ZAT zona 2 y e) ZAT zona 3.

Figure 6. Microstructures corresponding to diferent zones of a GMAW Hardox 400 joint: a) map of the zones, b) welding bead, c) HAZ zone 1, d) HAZ zone 2 and e) HAZ zone 3. 

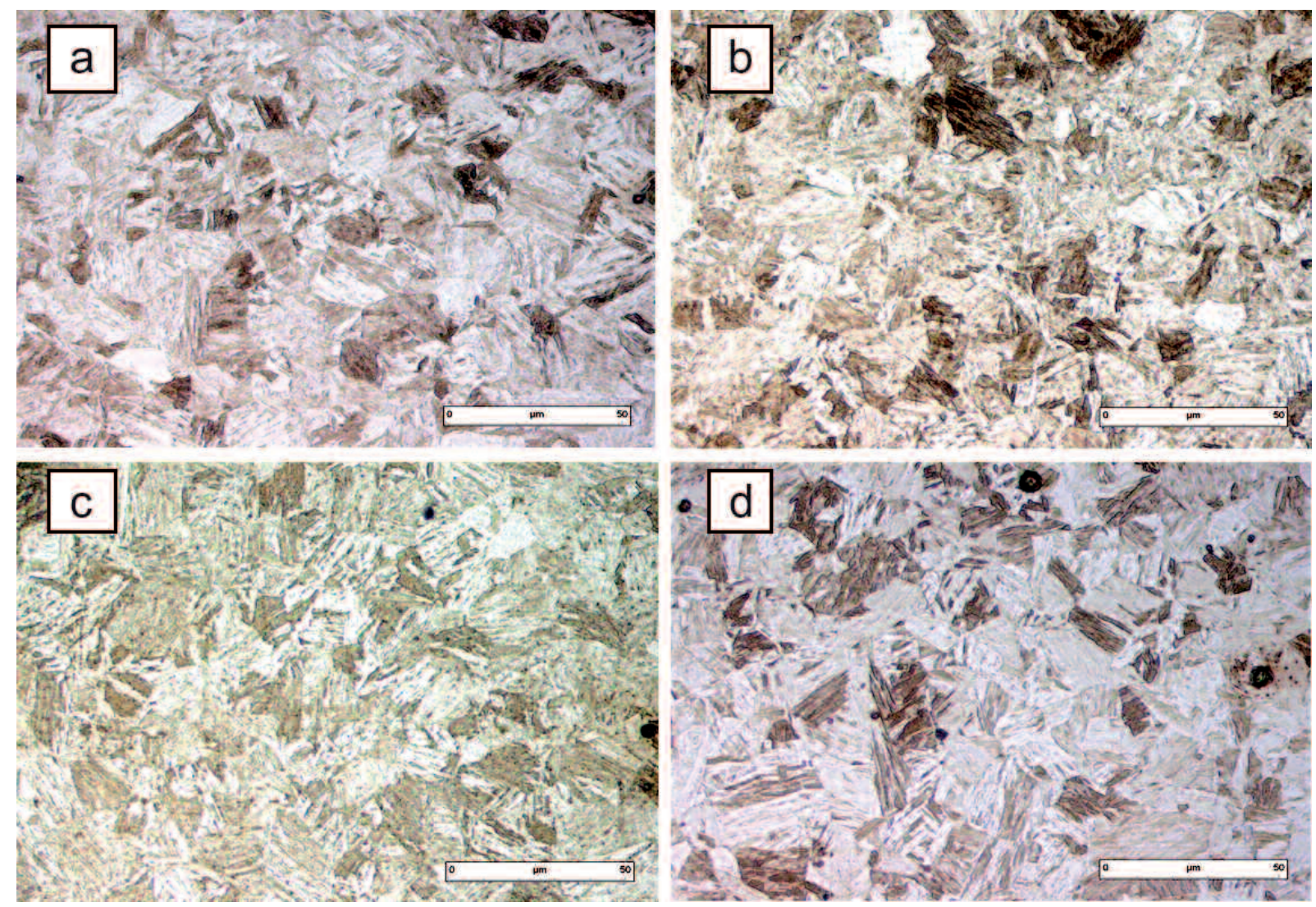

Figura 7. Microestructuras correspondientes a la ZAT (zona 4) para distintas condiciones de soldadura: a) soldadura en pasada única sin precalentamiento, b) soldadura en pasada única con precalentamiento, c) soldadura multipasada y d) zona 5: zona no afectada térmicamente.

Figure 7. Microstructures corresponding to the HAZ (zone 4) for several welding conditions: a) Only single pass welding, b) Only single pass welding with preheating, c) Multipass welding and d) zone 5: base metal.

El calentamiento alcanzado en la zona 2, inferior al de la zona 1, conduce a una austenización incompleta (Fig. 6 d)). Esta zona se caracteriza por la presencia de una estructura mixta de perlita FC y ferritas PF + FS(A) junto con martensita fuertemente revenida $\mathrm{M}$. En la probeta con precalentamiento, la zona 2 aparece algo más alejada del cordón, así como en la soldada en pasada múltiple.

La zona 3 se caracteriza por la presencia de martensita en láminas $\mathrm{M}(\mathrm{L})$ con un fuerte revenido (Fig. 6 e)). Del mismo modo que lo indicado anteriormente, aunque esta zona aparece en todos los modos de soldeo experimentados, se presenta a una distancia mayor del cordón de soldadura en las probetas precalentadas y en la soldada en varias pasadas.

La zona microestructural 4 (Fig. 7) presenta pequeñas diferencias en función del proceso de soldeo seguido, diferenciándose principalmente por la descomposición de la martensita $\mathrm{M}(\mathrm{L})$ debido el calentamiento. Así, las probetas soldadas sin precalentamiento, presentan una estructura muy similar a la del metal base, es decir, fundamentalmente martensita $\mathrm{M}(\mathrm{L})$, con características de revenido intermedio (Fig. 7 a)). En el caso de soldaduras efectuadas con precalentamiento, se observa lógicamente una descomposición mayor, debido a un calentamiento más alto (Figura 7 b)). En el caso de soldadura multipasada, la microestructura indica una estructura similar a la probeta con precalentamiento (Fig. 7 c)). Esto es congruente con el efecto esperado, de acuerdo a la valoración de áreas realizada en macroscopía.

La zona 5 o zona no afectada térmicamente (Fig. 7 d)) presenta estructura de martensita revenida $M(L)$.

\subsection{Ensayos de microdureza}

Los resultados obtenidos en los ensayos de microdureza se muestran en las figuras 8 y 9 . En la figura 8 se presentan los valores correspondientes a las probetas soldadas por una sola cara, con y sin precalenta- 


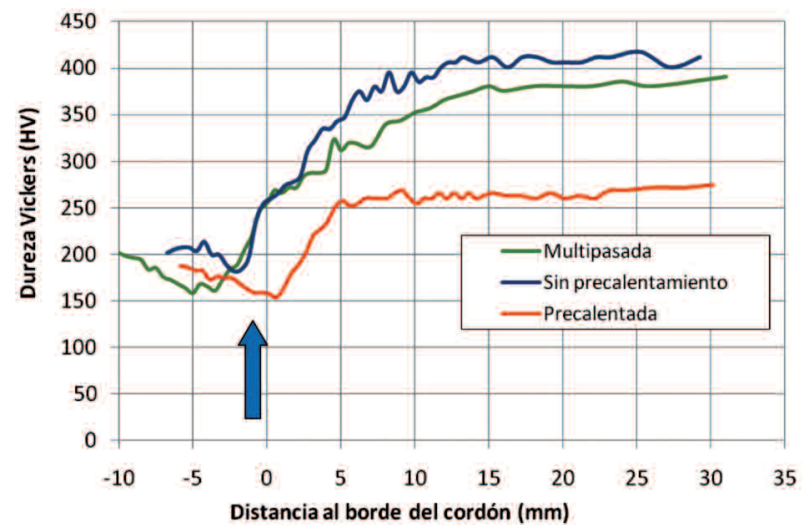

Figura 8. Resultados de microdureza de uniones soldadas por una sola cara con precalentamiento, sin precalentamiento y en multipasada.

Figure.8. Microhardness results of one side welding joints with preheating, without preheating

miento y en proceso multipasada. Como puede observarse, los valores mínimos de microdureza son similares en los tres procesos. En probetas sin precalentamiento y multipasada dicho valor mínimo de dureza se produce bajo el cordón, aunque cerca de su extremo lateral. En cambio, para las probetas precalentadas, el mínimo de dureza se halla situado justo en el borde del cordón, lo que facilitará la rotura.

Efectivamente, el borde del cordón puede actuar como un concentrador de tensiones debido al cambio de sección y a que la continuidad física entre el cordón y el metal base no siempre es perfecta. En ocasiones, pueden incluso aparecer defectos, como por ejemplo mordeduras, que potencian claramente la concentración de tensiones en el borde del cordón. Los valores obtenidos en los barridos realizados a diferente profundidad de la chapa son similares para cada proceso de soldadura, lo que indica que la chapa se ha homogeneizado térmicamente en la dirección del espesor durante el proceso de soldadura en todos los casos.

En la soldadura con precalentamiento la dureza de la zona no afectada no se recupera hasta una mayor distancia del cordón de soldadura, como era previsible.

Desde el punto de vista de la dureza, a pesar de lo enérgico del precalentamiento, no se observa ninguna ventaja respecto de la soldadura efectuada en frío ya que los valores mínimos de dureza alcanzados son similares en ambos casos.

La zona afectada térmicamente queda muy bien definida para la soldadura sin precalentamiento en una pasada y en multipasada. Lógicamente, en el proceso multipasada es mayor, como cabía esperar, ya

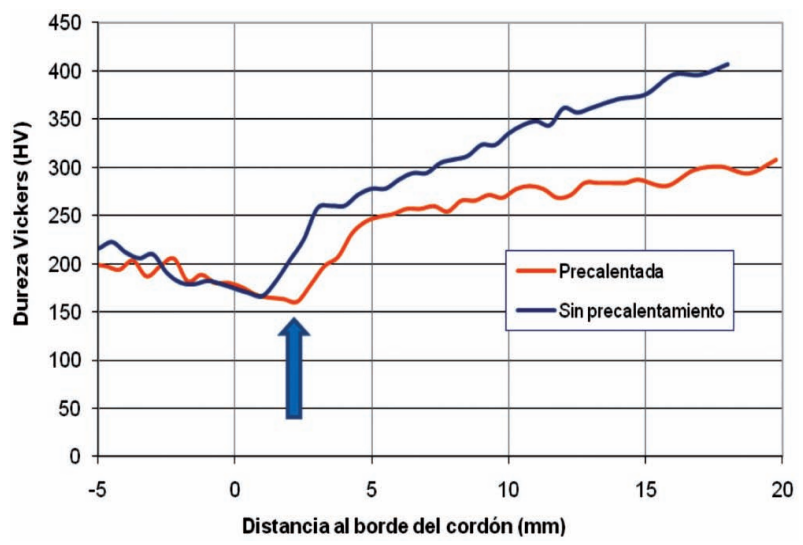

Figura 9. Resultados de microdureza de uniones soldadas por ambas caras, con precalentamiento y sin precalentamiento.

Figure 9. Microhardness results of both sides welding joints with preheating and without preheating.

que el volumen del cordón es casi 5 veces superior, en nuestro caso. Así mismo, se observa una pendiente intermedia en la recuperación de dureza respecto de los otros dos métodos, indicativo de que la zona afectada térmicamente a partir del extremo del cordón es también intermedia.

En cambio, en probetas soldadas por ambas caras, los resultados son distintos. En la figura 9 se representan los valores de dureza obtenidos en probetas soldadas por ambas caras. Como puede observarse la zona de mínimos de dureza se ve desplazada fuera del borde lateral del cordón, por lo que esta distribución presenta un comportamiento más adecuado frente a la rotura al separarse el efecto del borde del cordón y la zona de menor dureza. Esto es debido a que la soldadura en la primera cara actúa como precalentamiento local para la segunda. Al igual que para la soldadura en una cara, se amplía la zona afectada térmicamente respecto de la soldadura en frío, tal y como se apuntó anteriormente. La zona afectada térmicamente es, en cualquier caso mayor que la obtenida para la soldadura por una sola cara, tal y como puede comprobarse para la soldadura en frío en la que se alcanza una ZAT de $20 \mathrm{~mm}$ en el soldeo a doble cara frente a la de $12 \mathrm{~mm}$ obtenida en el caso de soldadura por una cara.

Este hecho es una consecuencia de que el segundo cordón de soldadura se ha efectuado sobre la chapa caliente, como se ha indicado anteriormente, actuando el primer cordón como sistema de un cierto precalentamiento del segundo y actuando aquél en el sentido indicado en el párrafo anterior y establecido en la figura 8 . 
Conviene indicar que con el precalentamiento se prevé un efecto beneficioso en relación al comportamiento a fatiga de la unión, si se tiene en cuenta el hecho de que en estas uniones la razón de heterogeneidad, MMR es menor de la unidad y, como se aprecia en la figura 9, el tratamiento de precalentamiento hace que ésta tienda a la unidad en la ZAT. En definitiva, se amplía la zona de la unión en la que se igualan las propiedades resistentes del material y del cordón, lo que de acuerdo a los trabajos de algunos autores ${ }^{[8 \mathrm{y} 9]}$, predice un mejor comportamiento a fatiga de la unión soldada.

\subsection{Ensayos de tracción}

Los ensayos de tracción fueron realizados sobre probetas soldadas por ambas caras ya que permiten abordar con ciertas garantías el ensayo dada la simetría axial requerida en el mismo. La temperatura ambiente durante los ensayos fue de $21^{\circ} \mathrm{C}$. Todas las probetas ensayadas rompieron justo por la zona de menor dureza de la ZAT, coincidiendo con el borde lateral del cordón de soldadura (Fig. 10). Los resultados obtenidos en los ensayos se establecen en la tabla III.

Como puede observarse, la resistencia a la tracción disminuye de manera importante en la chapa soldada, tanto si se practica precalentamiento como si se suelda en frío, alcanzándose disminuciones de hasta el $50 \%$ en algunos casos. Para tener una idea del comportamiento plástico de la zona de unión, se midió la estricción de rotura. Los resultados obtenidos se indican en la tabla IV.

La estricción es mayor en las probetas soldadas que en el material base. Los valores obtenidos para precalentamiento y en frío son similares, e incluso

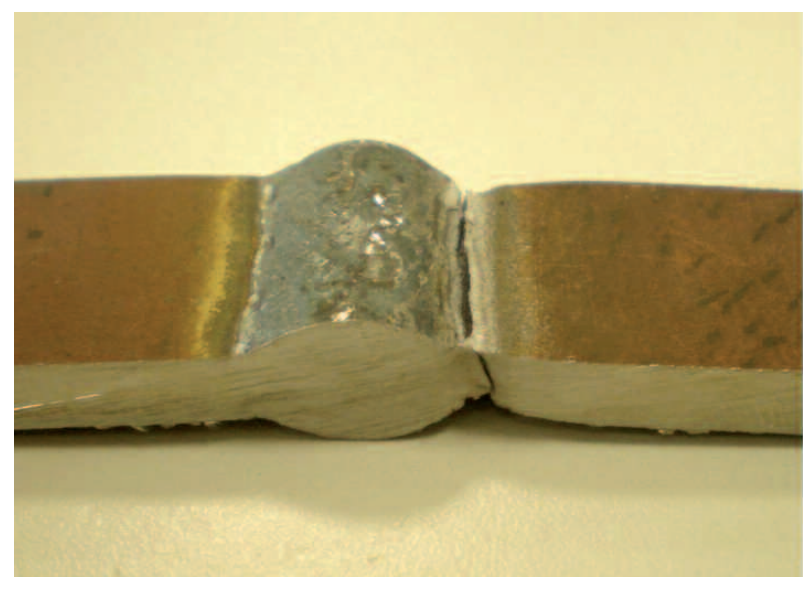

Figura 10. Detalle de la zona de rotura de una muestra sometida al ensayo de tracción (soldadura en dos caras).

Figure 10. Fracture detail of tensile sample (two sides welding).

mayores en el caso de las probetas soldadas con precalentamiento, lo que es consistente con la mayor zona afectada térmicamente que presentan.

\subsection{Ensayos de resiliencia}

En la tabla $\mathrm{V}$ se indican los valores de resiliencia obtenidos en las diferentes zonas de la ZAT para probetas soldadas por ambos lados con y sin precalentamiento. También se indican los valores correspondientes al metal base. La temperatura ambiente durante los ensayos fue de $21^{\circ} \mathrm{C}$. De los resultados obtenidos, se concluye que la tenacidad del material

Tabla III. Resistencia a la tracción de diferentes uniones soldadas

Table III. Tensile strength for welding joints

\begin{tabular}{|c|c|c|c|c|c|}
\hline $\begin{array}{l}\mathrm{N}^{\circ} \text { de } \\
\text { probeta }\end{array}$ & $\begin{array}{l}\text { Tipo de } \\
\text { probeta }\end{array}$ & $S_{o}\left(m^{2}\right)$ & $\begin{array}{c}\text { Carga } \\
\text { máxima } \\
\text { F }_{\text {m }}(k p)\end{array}$ & $\begin{array}{c}\text { Resistencia } \\
\text { a tracción } \\
R_{m}(\mathrm{MPa})\end{array}$ & $\begin{array}{c}\text { Valor } \\
\text { medio } \\
R_{m}(\mathrm{MPa})\end{array}$ \\
\hline 1 & Material & 118,11 & 15.708 & 1.305 & 1.313 \\
\hline 2 & Base & 91,05 & 12.269 & 1.322 & \\
\hline 3 & \multirow{3}{*}{ Sin Precal. } & 124,70 & 9.760 & 768 & \multirow[t]{3}{*}{696} \\
\hline 4 & & 124,67 & 8.307 & 654 & \\
\hline 5 & & 124,56 & 8.444 & 665 & \\
\hline 6 & \multirow[t]{3}{*}{ Con Precal. } & 124,92 & 8.071 & 634 & \multirow[t]{3}{*}{630} \\
\hline 7 & & 118,90 & 7.636 & 630 & \\
\hline 8 & & 118,70 & 7.568 & 625 & \\
\hline
\end{tabular}


INFLUENCIA DE LA TÉCNICA DE SOLDADURA MULTIPASADA Y DE LOS TRATAMIENTOS TÉRMICOS DE PRECALENTAMENTO Y POST-SOLDADURA EN EL COMPORTAMIENTO DE UNIONES GMAW DE UN ACERO MICROALEADO HARDOX 400 ANALISYS OF THE INFLUENCE OF THE MULTIPASS WELDING, WELDNG PREAHEAT AND WELDING POST HEAT TREATMENTS ON THE BEHAVIOUR OF GMAW JONTS OF HARDOX 400 MICROALLYYED STEEL

Tabla IV. Valores de estricción correspondientes a la rotura por tracción de uniones soldadas Table IV. Necking values obtained from the tensile tests of welding joints

\begin{tabular}{ccccccc}
\hline $\begin{array}{c}\mathbf{N}^{\circ} \text { de } \\
\text { probeta }\end{array}$ & $\begin{array}{c}\text { Tipo de } \\
\text { probeta }\end{array}$ & $\begin{array}{c}\mathbf{b}_{\mathbf{u}} \\
(\mathbf{m m})\end{array}$ & $\begin{array}{c}\mathbf{e}_{\mathbf{u}} \\
(\mathbf{m m})\end{array}$ & $\begin{array}{c}\mathbf{S}_{\mathbf{u}} \\
\left(\mathbf{m m}^{2}\right)\end{array}$ & $\begin{array}{c}\text { Estricción } \\
\text { de rotura } \mathbf{Z}(\%)\end{array}$ & $\begin{array}{c}\text { Valor medio } \\
\text { de } \mathbf{Z}(\%)\end{array}$ \\
\hline 1 & Material Base & 16,40 & 4,94 & 81,02 & 31,4 & 33,8 \\
2 & & 11,80 & 4,92 & 58,06 & 36,2 & \\
\hline 3 & Sin Precal. & 17,38 & 4,68 & 81,34 & 34,8 & 35,4 \\
4 & & 17,21 & 4,78 & 82,26 & 34,0 & \\
5 & & 17,33 & 4,50 & 77,99 & 37,4 & 52,8 \\
\hline 6 & Con Precal. & 17,46 & 3,40 & 59,36 & 52,5 & \\
\hline & & 16,99 & 3,35 & 56,92 & 52,1 & \\
\hline
\end{tabular}

es alta sea cual sea la forma en que se ha realizado la soldadura, con o sin precalentamiento.

En el ensayo se ha constatado sólo la rotura total únicamente en las probetas de material base libre de soldadura. En las restantes probetas ensayadas no se produce la rotura total de las mismas por lo que la energía absorbida en el impacto podría haber sido mayor (Fig. 11). De este modo, se puede reafirmar la mayor tenacidad observada en las probetas soldadas. Por otra parte, se aprecia que el ángulo de doblado de las diferentes probetas ensayadas y no rotas, esto es, para las probetas soldadas, es igual en todas ellas. Por tanto los valores de resiliencia obtenidos para estas últimas son comparables directamente. El aspecto de la rotura, de carácter más grisáceo y fibroso en las probetas soldadas constata lo

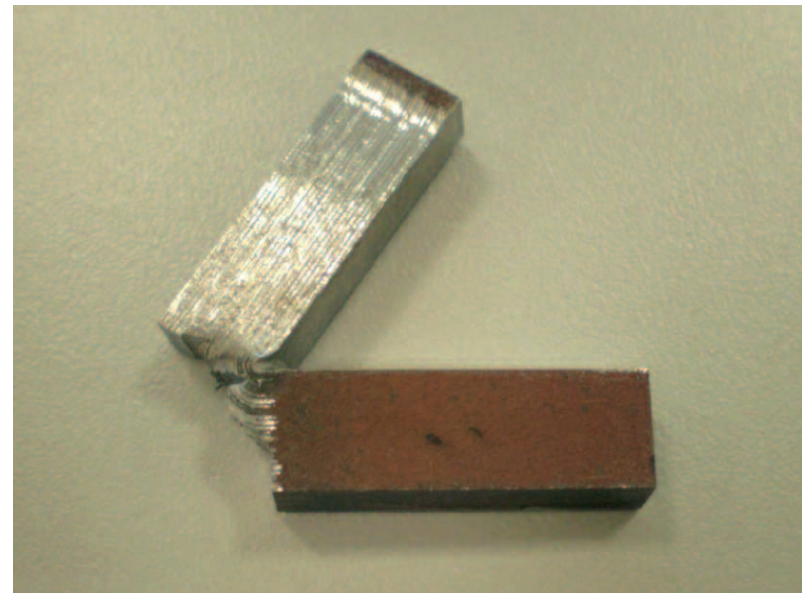

Figura 11. Probeta ensayada a resiliencia sin fractura total.

Figure 11. Tested resilience sample detail. indicado anteriormente en comparación con el metal base, cuya fractura es de carácter más frágil.

Por otra parte, analizando los resultados obtenidos en las probetas soldadas por ambas caras sin precalentamiento y con él, se puede apreciar un ligero incremento de la resiliencia en ambas, sin depender de la posición en la que se haya realizado la entalla en cada una de las probetas. En ambos grupos de probetas se obtiene un mismo valor de resiliencia correspondiente con la zona de entalla situada en el extremo del cordón de soldadura, pudiéndose considerar éste como un valor máximo en ambos casos. A partir del extremo del cordón, los valores de resiliencia se mantienen constantes para la soldadura con precalentamiento en tanto que en las probetas soldadas en frío la resiliencia tiende rápidamente hacia valores propios del metal base no afectado. Estos resultados son coherentes con los de dureza y con los microconstituyentes existentes en el acero en las diferentes zonas contempladas.

En la figura 12 se representan los resultados promediados para cada una de las zonas examinadas del material. Como puede observarse, la soldadura realizada con precalentamiento presenta un comportamiento más uniforme que la soldada en frío. Esta última refleja la tendencia típica inversa a los valores de dureza experimentados por el material. El límite inferior de resiliencia lo marca el material base.

\subsection{Influencia del tratamiento térmico postsoldeo}

Para comprobar el efecto de postcalentamientos en las uniones soldadas, se han realizado ensayos de tracción y microdureza a probetas sometidas a tratamientos térmicos postsoldeo a temperaturas similares a las 
Tabla V. Resultados de los ensayos de resiliencia de diferentes zonas de uniones soldadas

Table V. Resilience values for different welded joint areas

\begin{tabular}{|c|c|c|c|c|c|c|}
\hline $\begin{array}{l}\mathrm{N}^{\circ} \text { de } \\
\text { probeta }\end{array}$ & $\begin{array}{l}\text { Tipo de } \\
\text { probeta }\end{array}$ & $\begin{array}{l}\text { Posición } \\
\text { de Entalla }\end{array}$ & $\begin{array}{c}\text { Sección } \\
\text { rotura }\left(\mathrm{mm}^{2}\right)\end{array}$ & $\begin{array}{c}\text { Energía } \\
\mathrm{K}(\mathrm{J})\end{array}$ & $\begin{array}{l}\text { Resiliencia } \\
\rho(\mathrm{J} / \mathrm{mm} 2)\end{array}$ & $\begin{array}{l}\text { ¿Rotura? } \\
\text { (Si/No) }\end{array}$ \\
\hline 1 & Material & - & 32,04 & 53 & 1,65 & $S$ \\
\hline 2 & Base & - & 34,37 & 58 & 1,69 & $S$ \\
\hline 3 & \multirow{8}{*}{ Sin Precal. } & $\mathrm{CC}$ & 31,30 & 66 & 2,11 & $\mathrm{~N}$ \\
\hline 4 & & $\mathrm{CC}$ & 29,59 & 48 & 1,62 & $\mathrm{~N}$ \\
\hline 5 & & EC & 30,81 & 68 & 2,21 & $\mathrm{~N}$ \\
\hline 6 & & EC & 30,72 & 58 & 1,89 & $\mathrm{~N}$ \\
\hline 7 & & E1 & 32,60 & 60 & 1,84 & $\mathrm{~N}$ \\
\hline 8 & & E1 & 30,53 & 53 & 1,74 & $\mathrm{~N}$ \\
\hline 9 & & E2 & 32,91 & 56 & 1,70 & $\mathrm{~N}$ \\
\hline 10 & & E2 & 28,63 & 46 & 1,61 & $\mathrm{~N}$ \\
\hline 11 & \multirow{7}{*}{ Con Precal. } & $\mathrm{CC}$ & 35,29 & 70 & 1,98 & $\mathrm{~N}$ \\
\hline 12 & & $\mathrm{CC}$ & 31,72 & 62 & 1,95 & $\mathrm{~N}$ \\
\hline 13 & & EC & 30,08 & 56 & 1,86 & $\mathrm{~N}$ \\
\hline 14 & & EC & 32,39 & 72 & 2,22 & $\mathrm{~N}$ \\
\hline 15 & & E1 & 33,58 & 70 & 2,08 & $\mathrm{~N}$ \\
\hline 16 & & E1 & 30,95 & 62 & 2,00 & $\mathrm{~N}$ \\
\hline 17 & & E2 & 32,85 & 70 & 2,13 & $\mathrm{~N}$ \\
\hline
\end{tabular}

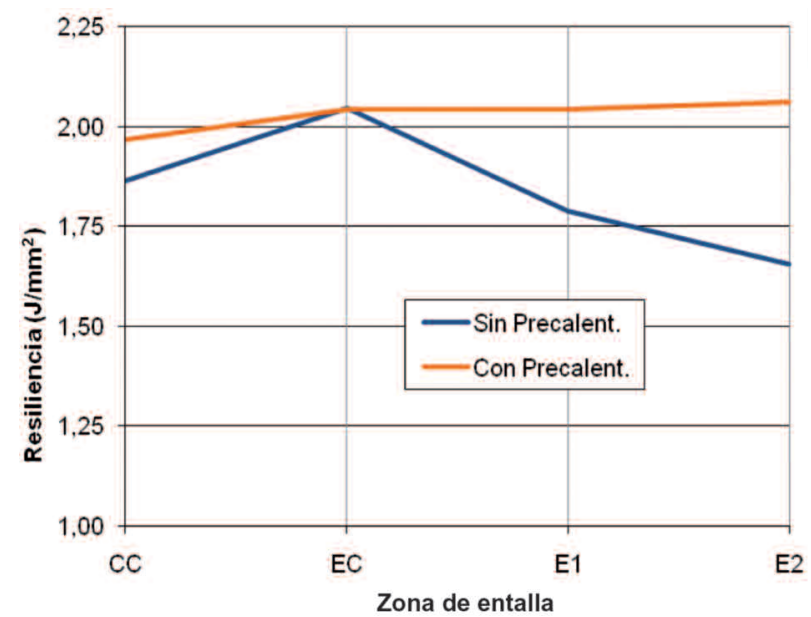

Figura 12. Valores de resiliencia (promediados) en función de la distancia al cordón de soldadura.

Figure 12. Resilience results (mean values) as a function of the distance to the welding bead.

utilizadas para precalentamientos, por su viabilidad de ejecución. Los tratamientos efectuados se especifican en la tabla VI. Los tratamientos térmicos han sido efectuados sobre probetas soldadas con y sin precalentamiento.
Tabla VI. Parámetros de los tratamientos postsoldeo

Table VI. Postwelding treatments parameters

\begin{tabular}{ccc}
\hline Tratamiento & Temperatura $\left({ }^{\circ} \mathrm{C}\right)$ & Tiempo (horas) \\
\hline TP1 & 150 & 2 \\
TP2 & 200 & 2 \\
TP3 & 250 & 2 \\
TP4 & 200 & 48 \\
\hline
\end{tabular}

Los resultados del comportamiento a tracción son los que aparecen en las tablas VII y VIII. Puede apreciarse que los resultados de resistencia a la tracción para postcalentamientos a las temperaturas elegidas son superiores a los obtenidos para probetas soldadas con o sin precalentamiento. Este resultado puede justificarse desde la óptica del alivio de tensiones internas que el postcalentamiento produce en el material.

Efectivamente, los tratamientos térmicos postsoldeo suelen conducir a la disminución de tensiones residuales en la soldadura de aceros por fusión, tal y como obtienen diversos autores ${ }^{[14-16]}$. La acción de las tensiones internas puede explicarse en base a 

ANALISYS OF THE INFLUENCE OF THE MULTIPASS WELDING, WELDING PREAHEAT AND WELDNG POST HEAT TREATMENTS ON THE BEHAVIOUR OF GMAW JONTS OF HARDOX 400 MICROALLOYED STEEL

Tabla VII. Valores resistentes de uniones sometidas a tratamientos postsoldeo

Table VII. Strength values of welded joints with postheating treatment

\begin{tabular}{|c|c|c|c|c|c|}
\hline $\begin{array}{l}\mathrm{N}^{\circ} \text { de } \\
\text { probeta }\end{array}$ & $\begin{array}{l}\text { Tipo de } \\
\text { probeta }\end{array}$ & $S_{o}\left(m^{2}\right)$ & $\begin{array}{l}\text { Carga máxima } \\
\qquad F_{m}(\mathrm{kp})\end{array}$ & $\begin{array}{c}\text { Resistencia a } \\
\text { tracción } R_{m}(\mathrm{MPa})\end{array}$ & $\begin{array}{l}\text { Valor medio } \\
\text { de } R_{m}(\mathrm{MPa})\end{array}$ \\
\hline $\begin{array}{r}9 \\
10\end{array}$ & $\begin{array}{l}\text { Postcalent. } \\
150^{\circ} \mathrm{C}-2 \mathrm{~h}\end{array}$ & $\begin{array}{l}120,12 \\
12157\end{array}$ & $\begin{array}{l}10.056 \\
10.061\end{array}$ & $\begin{array}{l}821 \\
812\end{array}$ & 817 \\
\hline $\begin{array}{l}11 \\
12\end{array}$ & $\begin{array}{l}\text { Postcalent. } \\
200^{\circ} \mathrm{C}-2 \mathrm{~h}\end{array}$ & $\begin{array}{l}120,50 \\
121,27\end{array}$ & $\begin{array}{r}10.076 \\
9.991\end{array}$ & $\begin{array}{l}820 \\
808\end{array}$ & 814 \\
\hline $\begin{array}{l}13 \\
14\end{array}$ & $\begin{array}{l}\text { Postcalent. } \\
250^{\circ} \mathrm{C}-2 \mathrm{~h}\end{array}$ & $\begin{array}{l}119,58 \\
120,08\end{array}$ & $\begin{array}{l}9.269 \\
9.753\end{array}$ & $\begin{array}{l}760 \\
797\end{array}$ & 779 \\
\hline $\begin{array}{l}15 \\
16\end{array}$ & $\begin{array}{l}\text { Postcalent. } \\
200^{\circ} \mathrm{C}-48 \mathrm{~h}\end{array}$ & $\begin{array}{l}118,56 \\
119,50\end{array}$ & $\begin{array}{l}9.937 \\
9.896\end{array}$ & $\begin{array}{l}822 \\
812\end{array}$ & 817 \\
\hline 17 & $\begin{array}{c}\text { Precal+Postcal } \\
200{ }^{\circ} \mathrm{C}-2 \mathrm{~h}\end{array}$ & 124,18 & 8.684 & 686 & 686 \\
\hline
\end{tabular}

Tabla VIII. Valores de estricción correspondientes a probetas de tracción de uniones sometidas a tratamientos térmicos postsoldeo

Table VIII. Necking values of tensile tested welded joints with postheating treatment

\begin{tabular}{|c|c|c|c|c|c|c|}
\hline $\begin{array}{l}N^{\circ} \text { de } \\
\text { probeta }\end{array}$ & $\begin{array}{l}\text { Tipo de } \\
\text { probeta }\end{array}$ & $\begin{array}{c}b_{u} \\
(m m)\end{array}$ & $\begin{array}{c}e_{u} \\
(\mathrm{~mm})\end{array}$ & $\underset{\left(\mathrm{mm}^{2}\right)}{\mathrm{S}_{\mathrm{u}^{2}}}$ & $\begin{array}{l}\text { Estricción de } \\
\text { rotura Z (\%) }\end{array}$ & $\begin{array}{c}\text { Valor medio } \\
\text { de Z (\%) }\end{array}$ \\
\hline 9 & Postcalent. & 16,78 & 4,29 & 72,0 & 40,1 & \multirow{2}{*}{40,3} \\
\hline 10 & $150^{\circ} \mathrm{C}-2 \mathrm{~h}$ & 16,41 & 4,41 & 72,4 & 40,5 & \\
\hline 11 & Postcalent. & 17,5 & 4,65 & 81,4 & 32,5 & \multirow{2}{*}{33,8} \\
\hline 12 & $200^{\circ} \mathrm{C}-2 \mathrm{~h}$ & 17,75 & 4,43 & 78,6 & 35,2 & \\
\hline 13 & Postcalent. & 17,39 & 4,47 & 77,7 & 35,0 & \multirow{2}{*}{33,7} \\
\hline 14 & $250^{\circ} \mathrm{C}-2 \mathrm{~h}$ & 17,41 & 4,66 & 81,1 & 32,4 & \\
\hline 15 & Postcalent. & 17,32 & 4,79 & 83,0 & 30,0 & \multirow{2}{*}{30,2} \\
\hline 16 & $200^{\circ} \mathrm{C}-48 \mathrm{~h}$ & 17,66 & 4,71 & 83,2 & 30,4 & \\
\hline 17 & $\begin{array}{c}\text { Precal+Postcal } \\
200^{\circ} \mathrm{C}-2 \mathrm{~h}\end{array}$ & 17,44 & 4,24 & 73,9 & 40,5 & 40,5 \\
\hline
\end{tabular}

modelos simplificados como el unidimensional ${ }^{[17]}$, de forma que la unión quede sometida a tensiones internas de tracción. En este caso, las tensiones disponibles para solicitaciones externas disminuirían y el alivio de tensiones conduce a mejorar la resistencia a la tracción del material. Existen experiencias que revelan la existencia de tensiones de tracción transversales al cordón en soldaduras similares y con espesores de acero igual al empleado en el presente trabajo $^{[18]}$ que permiten establecer como válido el modelo de comportamiento descrito. Resulta destacable que, en el caso del acero Hardox 400, no son 
aplicables tratamientos a alta temperatura y los tratamientos postsoldeo a bajas temperaturas producen resultados efectivos.

Así mismo, los valores de resiliencia son del mismo orden que los obtenidos para el material base o para probetas soldadas sin precalentamiento (Tabla V).

Los perfiles de microdurezas obtenidos para los postcalentamientos son concordantes con los resultados a tracción (Fig. 13). Puede apreciarse un ligero reforzamiento en la zona de mínima dureza de la ZAT, siendo el tratamiento más favorable el calentamiento a $200^{\circ} \mathrm{C}$ durante $2 \mathrm{~h}$. De esta forma, se puede afirmar que en las probetas soldadas sin precalentamiento sometidas a un determinado tratamiento térmico postsoldeo de relajación de tensiones se obtienen valores de resistencia a tracción algo mejores que en aquellas probetas que no han sido sometidas a ningún tipo de tratamiento térmico tras el proceso de soldadura.

La combinación de precalentamiento y postcalentamiento consigue una mezcla de ambos efectos: reforzamiento de la zona de mínima dureza a la vez que se amplía considerablemente la ZAT como consecuencia del precalentamiento.

\section{CONCLUSIONES}

- Como se ha establecido anteriormente, los dos parámetros que se han considerado con mayor influencia en la resistencia de una unión soldada en el acero objeto de estudio son la dureza y/o resistencia mínima y situación de la zona correspondiente en la unión soldada. El segundo se identifica con la amplitud de la zona afectada térmicamente.

- Ambos indicadores están directamente relacionados con la merma de características resistentes frente a la abrasión y a la fatiga, en relación al material base. En el cambio de sección entre el cordón y el material base concurren tanto la dureza mínima como la acumulación de tensiones. Además, la soldadura suele ejecutarse para la unión de los refuerzos estructurales a la chapa base. Esto se traduce en que esta zona se convierte en la más desfavorable para resistir los esfuerzos, ya que frente a sobreesfuerzos e impactos, toda la estructura se deformará elásticamente salvo la estrecha franja junto al cordón que será susceptible de deformación plástica por su menor límite elástico.

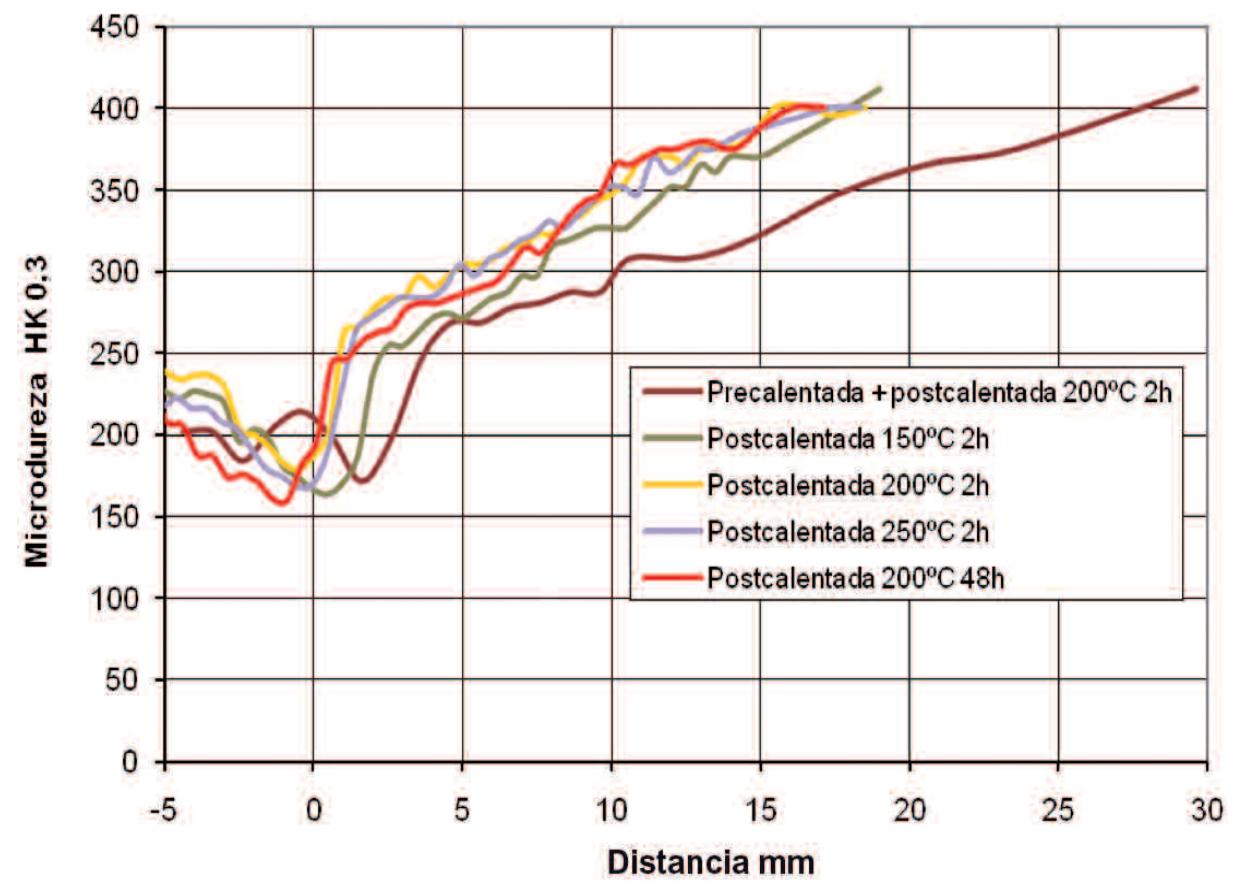

Figura 13. Comparación de la evolución de la microdureza para cada una de las probetas soldadas por ambas caras, sometidas a diferentes tratamientos térmicos de pre y post calentamiento.

Figure 13. Comparative analysis of the microhardness evolution for each both sides welding sample for different preheating and postheating treatments. 
- El beneficio de los tratamientos térmicos experimentados se justifica en base a los indicadores establecidos. Así los tratamientos térmicos postsoldeo mejoran la dureza de la zona afectada térmicamente. La resistencia de esta zona mejora en torno al $14 \%$ respecto al mismo proceso sin tratamiento como consecuencia de la eliminación de tensiones que tiene lugar. El precalentamiento amplía la zona afectada térmicamente consiguiendo que la zona adyacente al cordón pueda distribuir mejor la absorción de deformaciones. La combinación de ambos tratamientos consigue la unión de los dos efectos, por lo que resulta recomendable su ejecución siempre que sea posible.

- Además, el rediseño de los refuerzos puede ayudar en a la mejora de la distribución de tensiones y deformaciones plásticas. El alejamiento de la zona soldada de la rigidizada facilita una mejor adaptación a las solicitaciones.

\section{REFERENCIAS}

[1] X-H. Xue, Y-Y. Shan, L. Zheng y S-N. Lou, Mater. Sci. Eng. A 438-440 (2006) 285-287.

[2] M. Zrilic, V. Grabulov, Z. Burzic, M. Arsic y S. Sedmak, Int. J. Pres. Ves. Pip. 84 (2007) 139-150.

[3] R. Lagneborg, Mater. Des. 12 (1991) 3-14.

[4] P. Kulu, R. Veinthal, M. Saarna and R. Tarbe. Wear 263 (2007) 463-471.

[5] H.McI.Clark y R.J. Llewellyn. Wear 250 (2001) 32-44.

[6] SSAB, Ficha Técnica Hardox 400. 2007-0402. www.SSAB.com.

[7] S. Ravi, V. Balasubramanian, S. Babu y S. Nemat Nasser. Eng. Fail. Anal. 11 (2004) 619-634.

[8] S. Ravi, V. Balasubramanian, S. Babu y S. Nemat Nasser. Mater. Des. 25 (2004) 125-135.

[9] S. Ravi, V. Balasubramanian y S. Nemat Nasser. Int. J. Fatig. 27 (2005) 547-553.

[10] Welding Handbook, Welding Technology. American Welding Society, Eight Edition, Vol. 11.991, pp. 33-34.
[11] P.R. Sreenivasan, S.K. Ray, K.G. Samuel y S.L. Manna, Eng. Fract. Mech. 42 (1992) 1.0471.049 .

[12] T. Otarola, B. Bonnefois, M. Anglada, L. Coudreuse, y A. Mateo, Anales de Mecánica de la Fractura, Vol. II, Albarracín (Teruel, España), 2006, pp. 459-464.

[13] IIW / IIS, Welding in the World, Guidelines for the classification of ferritic steel weld metal. Vol. 24 (1986) N. 7/8, pp. 144-149.

[14] A.G. Olabi y M.S.J. Hashmi. J. Mat. Process. Tech. 55 (1995) 117-122.

[15] D. Tawfik, P.J. Mutton y W.K. Chiu. J. Mat. Process. Tech. 196 (2008) 279-291.

[16] A.M. Paradowska, J.W.H. Price, R. Ibrahim, T.R. Finlayson, M.I. Ripley y R. Blevins. J. Neutron Res. 1.477-2.655, Vol. 15, Issue 3 (2007) 231-241.

[17] Z. Feng, Processes and Mechanisms of welding residual stress and distortion, Ed. Woohdhead Publishing Limited, England, (2005) p. 8.

[18] P. Nevasmaa, Ph. Doctoral Thesis, Department of Mechanical Engineering, Oulu University, (2003).

\section{NOMENCLATURA}

$\mathrm{H}$ energía específica aportada, J/mm

P potencia total aportada, $\mathrm{W}$

v velocidad de soldeo, $\mathrm{mm} / \mathrm{s}$

$\mathrm{V}$ diferencia de potencial, $\mathrm{V}$

I intensidad de corriente, $\mathrm{A}$

f1 eficiencia de transferencia de energía a la pieza, adimensional

f2 eficiencia de fusión del proceso, adimensional

f3 eficiencia total del proceso, $\mathrm{mm}^{3} / \mathrm{J}$

Q energía específica de fusión del material, $\mathrm{J} / \mathrm{mm}^{3}$

Aw área total de la parte fundida, $\mathrm{mm}^{2}$

$\mathrm{K}_{\mathrm{c}}$ factor de intensidad de tensiones crítico

$\mathrm{CTOD}_{\mathrm{c}}$ valor de apertura de grieta crítico 